\title{
Driven fragmentation of granular gases
}

\author{
Raúl Cruz Hidalgo \\ AMADE, Departament de Física, Departament de Enginyeria Mecànica i de la Construcció Industrial, Universitat de Girona, \\ Avenida Montilivi s/n, 17071-Girona, Spain \\ Ignacio Pagonabarraga \\ Departament de Física Fonamental, Universitat de Barcelona Carrer Martí i Franqués, 1, 08028-Barcelona, Spain
}

(Received 5 March 2008; published 19 June 2008)

\begin{abstract}
The dynamics of homogeneously heated granular gases which fragment due to particle collisions is analyzed. We introduce a kinetic model which accounts for correlations induced at the grain collisions and analyze both the kinetics and relevant distribution functions these systems develop. The work combines analytical and numerical studies based on direct simulation Monte Carlo calculations. A broad family of fragmentation probabilities is considered, and its implications for the system kinetics are discussed. We show that generically these driven materials evolve asymptotically into a dynamical scaling regime. If the fragmentation probability tends to a constant, the grain number diverges at a finite time, leading to a shattering singularity. If the fragmentation probability vanishes, then the number of grains grows monotonously as a power law. We consider different homogeneous thermostats and show that the kinetics of these systems depends weakly on both the grain inelasticity and driving. We observe that fragmentation plays a relevant role in the shape of the velocity distribution of the particles. When the fragmentation is driven by local stochastic events, the long velocity tail is essentially exponential independently of the heating frequency and the breaking rule. However, for a Lowe-Andersen thermostat, numerical evidence strongly supports the conjecture that the scaled velocity distribution follows a generalized exponential behavior $f(c) \sim \exp \left(-c^{n}\right)$, with $n \approx 1.2$, regarding less the fragmentation mechanisms.
\end{abstract}

DOI: 10.1103/PhysRevE.77.061305

PACS number(s): 45.70.-n, 05.20.Dd

\section{INTRODUCTION}

Fragmentation is a phenomenon which arises commonly in many natural and technological processes [1-5]. As a result, the performance and quality of many technological activities are closely related to grain size control, which develops naturally in response to internal or external forcing. Consequently, understanding and controlling the complex phenomenology of fragmentation and the granular mixtures to which it gives rise has both a clear applied and fundamental interest.

There are cases where fragmentation is basically only sensitive to the grain properties. In this limiting situation, which may arise when the external driving becomes dominant, the fragmentation process does not depend significantly on particle collisions and the fragmentation dynamics is linear $[6,7]$. However, for granular materials usually the interaction between the grains plays a relevant role and the evolution is nonlinear. Such scenarios include a wide variety of externally driven situations, such as granulate processing [1], particle comminution [2], shattering of solid objects [4], or meteorite clouds [5]. Theoretical studies on nonlinear fragmentation have been carried out, following mean-field theory [6-8], which neglect the role of particle velocities at impact and describe the evolution of the particle size distribution. These approaches to linear and nonlinear fragmentation have led to explicit results, providing insight into the emergence of dynamic scaling regimes [6,7,9-11].

In order to gain understanding of the physics of fragmentation, during the past decades, quantitative experimental studies have been carried out to analyze systematically the physical processes involved in collision-induced fragmenta- tion $[4,12,13]$. Early studies on the fragmentation of brittle glass spheres smashed against a wall [12] showed that the glass breaks into pieces of a wide range of sizes, which can be characterized by a power-law mass distribution with a characteristic exponent $\frac{2}{3}$. Although generically wide mass distributions are obtained, Ishii and Matsushita [13] showed that particle size distributions change from a log-normal to a power-law distribution as the falling height increases. Following the same procedure, but using thick plates of dry clay, Meibom and Balslev [14] figured out that the mass distribution of fragments presents a crossover between two different power-law regimes also with remarkable size dependence. Further studies on a variety of materials have led to the conclusion that size distributions vary significantly and that they are more sensitive to the material properties rather than to the particle shape [4].

Computer simulations have also been used to study the explosive fragmentation of particle clusters. Starting from a thermalized cluster, this linear fragmentation process leads to a wide distribution of fragment sizes, which was initially characterized as an algebraic distribution with an exponent $-1[15,16]$. More recent studies have shown that the cumulative fragment distribution is better described by the scaling ansatz $P(m) \sim m^{-\alpha} \exp \left[-\left(m / m_{0}\right)^{\alpha}\right]$, where $m$ is the fragment mass, $m_{0}$ is a characteristic mass, and $\alpha$ is a nonuniversal scaling exponent which depends on the temperature [16]. Collision-induced fragmentation has also been explored through computer simulations $[17,18]$. These studies have shown that also these systems evolve into a mixture characterized by power-law or log-normal fragment size distribution depending on the details of the fragmentation process [18]. 
There has not been much effort devoted to the analysis of the collective properties of granular materials emerging as a result of collision-induced grain fragmentation. In comminution, computer simulations have shown the development of force chain networks and the role of their spatial distribution of the comminution efficiency in ball mills [17]. For homogeneous granular gases, earlier studies have focused on their kinetics and have characterized the corresponding dynamical regimes $[11,19]$. We want to extend previous results and analyze the kinetic evolution of granular gases when energy is injected homogeneously. The careful and systematic study of such simplified scenarios will help to clarify the relative importance of the competing processes which determine the dynamic evolution of granular materials. Since fragmentation is related to the collisions suffered by the grains, one needs to account both for the grain velocity and size as well as for grain inelasticity and the energy lost at each collision. Previous studies have shown that the material properties and the mechanics of fragmentation affect the grain size distribution. Therefore, rather than concentrating on a realistic modeling of fragmentation for a particular material, we will describe the breakup process of grains as a stochastic process and consider a wide family of fragmentation events. We are interested in understanding which features of such processes become relevant in the material collective response, which can be better addressed from a more general framework. It is well known that for these driven systems some properties depend on the details of energy injection. To assess the role of such a mechanism in the fragmentation kinetics, we consider some previously proposed thermostats [20-23]. We implement two types of momentum-conserving thermostats, which allow a more faithful description of the intrinsic dynamics, and describe which features depend on the particular thermostat under scrutiny.

We introduce the model in Sec. II. In Sec. III we analyze the kinetic evolution of the granular gas analytically in the asymptotic dynamic scaling regime. Such a study sets already the role of the fragmentation probability and its interrelation with grain number. In Sec. IV we describe the numerical method, based on direct simulation Monte Carlo (DSMC) calculations, which allows us to verify the hypothesis of dynamic scaling and provides us with the size and velocity distributions these granular gases develop as a result of their intrinsic dynamics. We report the main results of this numerical model in Sec. V and conclude with a summary of the main results and perspectives.

\section{MODEL}

We consider a gas of spherical particles of radius $\sigma$ which interact through hard-core potentials; therefore, all collisions are binary and instantaneous. For a $D$-dimensional system, at each collision a fraction $\left(1-\alpha^{2}\right) /(2 D)$ of the relative kinetic energy is lost, where $\alpha \leq 1$ is the restitution coefficient ( $\alpha$ $=1$ corresponds to completely elastic particles). Since energy is not conserved for $\alpha<1$, a freely evolving gas will eventually be arrested. Energy must be injected to reach a steady state; hence, in theses systems the steady-state features are intimately related to the energy injection mechanisms. We will concentrate in the simplest case where energy input is homogeneous, leading to homogeneous steady states.

Since grains are extended objects, they will develop defects as a result of their subsequent collisions, and defect buildup leads eventually to particle breakup. During grain collisions the relative kinetic energy of the incoming colliding pair will be stored transiently as elastic potential energy $E_{p}$ and a part of it will be recovered as kinetic energy. The details of this process and the corresponding particle deformations will determine how defects develop and lead to particle fragmentation. Experimental studies on the collisions of particles against large obstacles have shown that very brittle materials break if the stored potential energy becomes larger than a characteristic threshold, while soft particles will typically accumulate energy dissipation on a number of collisions, leading to damage evolution and defect growth, before breaking up [24]. Detailed theoretical studies which address how energy is stored and dissipated during the interaction between grains and their dependence on grain deformation will be extremely helpful to gain detailed insight into the origin and propagation of particle defect generation [25].

Rather than describing in detail how grains deform and store energy during their interaction, we will regard collisions as instantaneous and use the relative incoming kinetic energy of the grain pair as a measure of the stored potential energy. Due to the intrinsic uncertainty of grain breakup, we then adopt a statistical description of the fragmentation event. To this end, we use the stored energy $E_{p}$ and grain size to determine the breaking probability of the colliding particles; hence, the fragmentation can be regarded as a stochastic process. Since we do not keep memory of the collision events of the grains, this approach corresponds to brittle materials, although the generalization to soft particles is straightforward.

The larger the accumulated potential energy at a collision, $E_{p}$, the larger the chance that grain defects increase, leading to grain breakup. Since the number of defects grows with grain area, a usual form for the breaking probability is given by the Weibull distribution [26]; we generalize it to a fragmentation probability of the form

$$
P\left(\sigma, E_{p}\right)=1-\exp \left(-A_{0} \sigma^{\gamma} E_{p}^{\rho}\right),
$$

where $A_{0}$ is a material parameter which characterizes the relevant size and energy scales involved in particle fragmentation [26]. The Weibull distribution corresponds to the particular case $\gamma=D-1$ and $\rho=0$. The exponents $\gamma$ and $\rho$ may be either positive or negative. If their combination leads to a decrease of the product $\sigma^{\gamma} E_{p}^{\rho}$, then typically the fragmentation probability will decrease with time. On the contrary, an increasing product will lead to an effective constant fragmentation probability after a transient and the fragmentation process will become independent of the details of the colliding grains. In all cases, the model we will analyze differs from standard fragmentation models based on the mean-field fragmentation equation which do not correlate particle fragmentation with grain collisions [6].

To complete the description of fragmentation, we have to specify the number, size, shape, and velocities of outgoing 
particle fragments and how they are related to the broken grain. Here the situation is more controversial, and in general it depends on the mechanisms which drives fragmentation. For collision-induced fragmentation, the Roslin-Rammer law is commonly used [6]. In the present work, for simplicity's sake we will assume that a fragmenting grain breaks into two offsprings which will keep the spherical shape of the parent. The mass of the parent is conserved, and it is distributed uniformly between the two offsprings. The fragments also inherit the parent velocity, and we will disregard energy dissipation during the fragmentation process.

If we neglect the role of fluctuations and concentrate on the mean behavior of the system, the homogeneous Boltzmann equation will determine the time evolution of homogeneous granular gases through the temporal evolution of the one-particle distribution function $f(\mathbf{v}, \sigma, t)$, where $\mathbf{v}$ stands for the particle velocity. In a $D$-dimensional space this equation reads

$$
\begin{aligned}
\frac{\partial f(\mathbf{v}, \sigma, t)}{\partial t}= & \frac{1}{2} \int d u g(u) \int d \mathbf{v}_{1} d \mathbf{v}_{2} d \sigma_{1} d \sigma_{2} d \hat{\varepsilon} \theta\left(\mathbf{v}_{12} \cdot \hat{\varepsilon}\right)\left|\mathbf{v}_{12} \cdot \hat{\varepsilon}\right| \sigma_{12}^{D-1}\left(f ( \mathbf { v } _ { 1 } , \sigma _ { 1 } , t ) f ( \mathbf { v } _ { 2 } , \sigma _ { 2 } , t ) \left\{-\delta\left(\mathbf{v}-\mathbf{v}_{1}\right) \delta\left(\sigma-\sigma_{1}\right)\right.\right. \\
& -\delta\left(\mathbf{v}-\mathbf{v}_{2}\right) \delta\left(\sigma-\sigma_{2}\right)+\left[1-P\left(\sigma_{1}, E_{p 1}\right)\right] \delta\left(\mathbf{v}-\mathbf{v}_{1}^{*}\right) \delta\left(\sigma-\sigma_{1}\right)+\left[1-P\left(\sigma_{2}, E_{p 2}\right)\right] \delta\left(\mathbf{v}-\mathbf{v}_{2}^{*}\right) \delta\left(\sigma-\sigma_{2}\right) \\
& +P\left(\sigma_{1}, E_{p 1}\right) \delta\left(\mathbf{v}-\mathbf{v}_{1}^{*}\right)\left[\delta\left(\sigma-\sigma_{1} \cdot u^{1 / D}\right)+\delta\left(\sigma-\sigma_{1} \cdot(1-u)^{1 / D}\right)\right]+P\left(\sigma_{2}, E_{p 2}\right) \delta\left(\mathbf{v}-\mathbf{v}_{2}^{*}\right)\left[\delta\left(\sigma-\sigma_{2} \cdot u^{1 / D}\right)\right. \\
& \left.\left.\left.+\delta\left(\sigma-\sigma_{2} \cdot(1-u)^{1 / D}\right)\right]\right\}\right)+F f(\mathbf{v}, \sigma, t)
\end{aligned}
$$

where $\hat{\varepsilon}$ is a unit vector joining the centers of the colliding grains. The Heaviside function $\theta$ ensures the appropriate kinematic constraint that only approaching grains will collide, while $\left|\mathbf{v}_{12} \cdot \hat{\varepsilon}\right| \sigma_{12}^{D-1}$ accounts for the collision cross section. In the previous equation $\mathbf{v}_{12}=\mathbf{v}_{1}-\mathbf{v}_{2}$ and $\sigma_{12}=\left(\sigma_{1}+\sigma_{2}\right) / 2$, while $g(u)$ corresponds to the probability density that one of the offsprings, in the case of fragmentation, has a mass $u$ times the parent mass. Mass conservation implies $g(u)=g(1-u)$. In the model we will analyze subsequently, we will concentrate in the simplest case where this distribution is uniform-i.e., $g(u)=1$ and $0 \leq u \leq 1$.

The first two terms on the right-hand side of Eq. (2) account for the disappearance of particles of velocity $\mathbf{v}$ due to collisions. Through collisions, particles with velocity $\mathbf{v}$ are also generated from grains with precollisional velocities $\mathbf{v}^{*}$. It is through these precollisional velocities that the inelastic character of the collision enters through the inelasticity parameter $\alpha$. Indeed, the precollision velocities $\mathbf{v}_{1}^{*}$ and $\mathbf{v}_{2}^{*}$, in terms of the post-collisional ones $\mathbf{v}_{2}$ and $\mathbf{v}_{2}$ (the relevant velocities to describe the kinetic evolution), are given by

$$
\begin{aligned}
& \mathbf{v}_{1}^{*}=\mathbf{v}_{1}-\frac{m_{2}}{m_{1}+m_{2}}\left(1+\alpha^{-1}\right)\left[\hat{\varepsilon} \cdot \mathbf{v}_{12}\right] \hat{\varepsilon}, \\
& \mathbf{v}_{2}^{*}=\mathbf{v}_{2}+\frac{m_{1}}{m_{1}+m_{2}}\left(1+\alpha^{-1}\right)\left[\hat{\varepsilon} \cdot \mathbf{v}_{12}\right] \hat{\varepsilon},
\end{aligned}
$$

The two terms in Eq. (2) containing the prefactor 1 $-P\left(\sigma, E_{p i}\right)$ correspond to collisions in which, with probability $1-P\left(\sigma, E_{p i}\right)$, particles do not break. The last four terms account for the fragmentation of grain 1 with mass $m_{1}$ ( $=\sigma_{1}^{D}$ ) [grain 2 with mass $m_{2}$ ], leading to the creation of two offsprings with masses $u m_{1}$ and $(1-u) m_{1}\left[u m_{2}\right.$ and (1 $-u) m_{2}$. Mass conservation leads to correlation between the sizes of the newborn particles.
The last term in the Boltzmann equation corresponds to an external forcing which injects energy in the gas. In its absence, for $\alpha<1$, the system cools, leading asymptotically to a system at rest. Energy is usually introduced inhomogeneously through the boundaries. However, we will concentrate on homogeneous systems and will study homogeneous thermostats. Different one-particle thermostats have been proposed in the literature [23]; we identify them generically as $F f(\mathbf{v}, \sigma, t)$ and will analyze some of them in more detail in subsequent sections.

In order to gain insight into the nature of the fragmentation of inelastic granular gases, we will consider in Sec. III the kinetic evolution of averaged quantities for which it is possible to carry out a detailed analytical study. In Sec. IV we describe the numerical method we will use subsequently in Sec. $\mathrm{V}$ to validate the theoretical predictions and analyze the grain size and velocity distributions in the dynamical scaling regime. We conclude in Sec. VI with a discussion of the main results derived and their implications for the kinetics of fragmenting gases.

\section{KINETICS}

The kinetics of homogeneous fragmenting systems can be addressed through the study of moments of the distribution function, $M_{n m}(t) \equiv \int_{0}^{\infty} d \sigma \int_{-\infty}^{\infty} d \mathbf{v} \sigma^{n} v^{m} f(\mathbf{v}, \sigma, t)$, in the framework of the Boltzmann equation. For example, mass conservation implies that $M=M_{D 0}$ does not evolve in time, as can be easily verified from Eq. (2). The total number of grains, $N(t) \equiv M_{00}(t)$, increases monotonically in time. Its evolution can be derived again from Eq. (2), leading to

$$
\frac{d N}{d t}=\tilde{\omega}(t) N(t)
$$

where $\widetilde{\omega}(t)$ is the fragmentation frequency, given by 


$$
\begin{aligned}
\widetilde{\omega}(t)= & \frac{1}{2 N(t)} \int d \hat{\varepsilon} d 1 d 2 \sigma_{12}^{D-1} \theta\left(\hat{\varepsilon} \cdot \mathbf{v}_{12}\right) \\
& \times\left|\hat{\varepsilon} \cdot \mathbf{v}_{12}\right| f\left(\mathbf{v}_{2}, \sigma_{2}, t\right) f\left(\mathbf{v}_{1}, \sigma_{1}, t\right)\left[P\left(\sigma_{1}, E p_{1}\right)\right. \\
& \left.+P\left(\sigma_{2}, E p_{2}\right)\right] .
\end{aligned}
$$

The shorthand notation $d 1 d 2$ stands for $d \mathbf{v}_{1} d \sigma_{1} d \mathbf{v}_{2} d \sigma_{2}$, and $P\left(\sigma_{1}, E p_{1}\right)$, the breaking probability, makes it explicit that the number of grains increases only due to fragmentation.

For inelastic grains, the global energy $E(t) \equiv M_{D 2}(t)$ is not conserved and evolves according to

$$
\frac{d E}{d t}=-b_{c}(t) \omega(t) \gamma_{0} E+\xi_{0}^{2} \psi
$$

where $\gamma_{0}=\frac{1-\alpha^{2}}{2 D}$ and $b_{c}$ is the dimensionless collisional average given by

$$
\begin{aligned}
b_{c}= & \frac{D}{2 \omega E_{c}} \int d \hat{\varepsilon} d 1 d 2 \theta\left(\mathbf{v}_{12} \cdot \hat{\varepsilon}\right) \\
& \times\left|\mathbf{v}_{12} \cdot \hat{\varepsilon}\right|^{3} \frac{m_{1} m_{2}}{m_{1}+m_{2}} f\left(\mathbf{v}_{1}, \sigma_{1}, t\right) f\left(\mathbf{v}_{2}, \sigma_{2}, t\right),
\end{aligned}
$$

and $\omega$ is the collision frequency, which reads as

$$
\begin{aligned}
\omega(t)= & \frac{1}{N(t)} \int d \hat{\varepsilon} d 1 d 2 \sigma_{12}^{D-1} \theta\left(\hat{\varepsilon} \cdot \mathbf{v}_{12}\right) \\
& \times\left|\hat{\varepsilon} \cdot \mathbf{v}_{12}\right| f\left(\mathbf{v}_{1}, \sigma_{1}, t\right) f\left(\mathbf{v}_{2}, \sigma_{2}, t\right) .
\end{aligned}
$$

For constant fragmentation probability $P\left(\sigma, E_{p}\right)=p_{0}$, the fragmentation and collision frequencies are directly proportional to each other, $\widetilde{\omega}(t)=p_{0} \omega(t)$, showing the close correlation between collision and fragmentation events. The last term in Eq. (7) accounts for energy injection through a homogeneous thermostat. It does not appear in Eq. (5) as the thermostat conserves the number of particles. The factor $\xi_{0}$ characterizes the magnitude of the energy input (a freely evolving granular gas corresponds to the limiting case $\xi_{0}$ $=0$ when no thermostat acts on the system [11]), while the specific type of thermostat will determine the functional form of $\psi$. The kinetic evolution of fragmenting gases is determined by the dependence of the thermostat on the collision frequency and hence on how $\psi$ depends on $\omega(t)$. In Sec. V we will discuss the relevance of the energy injection mechanism on size and velocity grain distributions.

One can make further analytic progress assuming that after a transient the material relaxes into a dynamic scaling regime described by a unique characteristic velocity $\bar{v}(t)$ and size $\bar{r}(t)$. In this scaling regime, the time dependence enters only through the time evolution of these characteristic quantities. Accordingly, the time dependence of the distribution function reduces to

$$
f(\mathbf{v}, \sigma, t) \propto \frac{N(t)}{\bar{r} \bar{v}^{D}} \widetilde{f}(\mathbf{c}, \bar{\sigma}),
$$

where we have introduced the rescaled size and velocity

$$
\mathbf{c}=\frac{\mathbf{v}}{\bar{v}(t)}, \quad \bar{\sigma}=\frac{\sigma}{\bar{r}(t)} .
$$

A natural choice for the characteristic velocity $\bar{v}$ is the variance of the velocity distribution $\bar{v}^{2}=\left\langle v^{2}\right\rangle$, while for the characteristic length $\bar{r}$, we take the mean particle radius $\bar{r}=\langle\sigma\rangle$. The dependence on $N(t)$ comes from the normalization condition. Mass conservation implies that the mass for each particle, $m$, scales as $m \sim 1 / N$. Hence the characteristic size decreases as $\bar{\sigma} \propto N^{-1 / D}$. As a result, the collision frequency evolves as

$$
\omega(t) \propto \widetilde{v} N(t) \bar{\sigma}^{D-1}=\widetilde{v} N(t)^{1 / D} .
$$

In the dynamic scaling regime, $\tilde{f}(\mathbf{c}, \bar{\sigma})$ becomes a normalized time-independent distribution which shows that in this regime the relative amounts of the different species which evolve internally through the fragmentation do not change in time. Dynamic scaling has been shown to hold on different types of fragmenting systems [11,27]; we will analyze its implications assuming dynamic scaling holds and will check its validity in the next sections.

In order to study the time evolution of a fragmenting gas, it is useful to consider an intrinsic time scale $\mathcal{C}$ directly related to the number of collisions suffered by a particle [28]:

$$
d \mathcal{C}=\omega d t .
$$

In terms of this new time scale, Eq. (5) can be expressed as $d N / d \mathcal{C}=N(t) \widetilde{\omega}(t) / \omega(t)$. Accordingly, the particle number evolution in the scaling regime reduces to

$$
\frac{d N}{d \mathcal{C}} \sim P\left(\bar{\sigma}(t), \bar{E}_{p}(t)\right) N(t),
$$

where $\bar{E}_{p}(t)$ will be a function of $\bar{\sigma}$ and $\bar{v}$. In the scaling regime, the generalized Weibull breaking probability relaxes either to a constant $p_{0}$ or vanishes as a power law, $P\left(\sigma, E_{p}\right) \sim \sigma^{\gamma} E_{p}^{\rho}$. Therefore, the results we will describe are in fact applicable to any fragmentation probability with asymptotic algebraic behavior. Since $E_{p}$ is of the order of the kinetic energy that a particle carries during a collision, $E_{p}$ $\sim m \bar{v}^{2} \sim \bar{v}^{2} / N$, the fragmentation probability reduces to

$$
P\left(\bar{\sigma}, \bar{E}_{p}\right) \propto \bar{v}^{2 \rho} \bar{\sigma}^{\gamma} N^{-\rho} \propto \bar{v}^{2 \rho} N^{-D \rho+\gamma / D} .
$$

After substituting Eq. (15) into Eq. (6), we also obtain the time evolution of the fragmentation frequency:

$$
\widetilde{\omega}(t) \propto \bar{v} N \bar{\sigma}^{D-1} P\left(\bar{\sigma}, \bar{E}_{p}\right) \propto \bar{v}^{2 \rho+1} N^{1-\rho D-\gamma / D} .
$$

We will now analyze the different kinetic scenarios associated to the thermostat heating frequency.

\section{A. Freely evolving granular gas}

Let us start by considering the evolution of a freely evolving gas, with a size-dependent breaking probability. This limiting situation corresponds to $\mathcal{F} f(\mathbf{v}, \sigma, t)=0$ in the Boltzmann equation, when no energy is injected. In this case, the energy evolution equation reduces to 


$$
\frac{d E}{d \mathcal{C}}=-2 b_{c} \gamma_{0} N E
$$

yielding

$$
E=E_{0} e^{-2 b_{c} \gamma_{o} \mathcal{C}}
$$

where $E_{0}$ is a characteristic energy scale in the scaling regime. The total energy is related to the typical particle velocity because in the scaling regime $E(t) \propto N \bar{\sigma}^{D} \bar{v}^{2} \propto \bar{v}^{2}$. Hence, substituting Eqs. (18) and (15) into Eq. (14) leads to

$$
\frac{d N}{d \mathcal{C}}=N^{1-\gamma / D-\rho} E_{0}^{\rho} e^{-2 b_{c} \gamma_{0} \rho \mathcal{C}} .
$$

There are different kinetic scenarios with qualitative variations in the time evolution of the number of grains depending on the temporal evolution of the fragmentation probability

\section{Constant fragmentation probability}

This is the simpler case, which corresponds to $\gamma=\rho=0$ and has been analyzed previously [11]. In this scenario the breaking probability is $p_{0}$ and the time evolution of the number of grains reads simply

$$
N=N_{0} e^{p_{0} \mathcal{C}},
$$

where $N_{0}$ is the initial number of grains in the scaling regime. In order to obtain the explicit time dependence, we need to solve Eq. (13). Using the relation between collision frequency and particle number, one arrives at

$$
\frac{d \mathcal{C}}{d t}=N_{0}^{1 / D} E_{0} e^{-b_{c} \gamma_{0} \mathcal{C}+\mathcal{C} / D},
$$

which leads to

$$
\mathcal{C}=\frac{1}{b_{c} \gamma_{0}-1 / D} \ln \left[1+\left(b_{c} \gamma_{0}-\frac{1}{D}\right) E_{0}^{1 / 2} N_{0}^{1 / 2}\left(t-t_{0}\right)\right] .
$$

Accordingly, the time evolution of the number of grains and energy reduces to

$$
\begin{aligned}
& N=N_{0}\left[1+\left(b_{c} \gamma_{0}-\frac{1}{D}\right) E_{0}^{1 / 2} N_{0}^{1 / 2}\left(t-t_{0}\right)\right]^{1 / b_{c} \gamma_{0}-p_{0} / D}, \\
& E=E_{0} \frac{1}{\left[1+\left(b_{c} \gamma_{0}-\frac{1}{D}\right) E_{0}^{1 / 2} N_{0}^{1 / 2}\left(t-t_{0}\right)\right]^{2 b_{c} \gamma_{0} / b_{c} \gamma_{0}-p_{0} / D}} .
\end{aligned}
$$

In the absence of fragmentation, $p_{0}=0$, the number of particles does not increase and the energy vanishes as $E(t)$ $\sim t^{-2}$, which corresponds to the well-known Haff's law [29]. Hence, Eq. (24) can be regarded as a generalization of Haff's law for fragmenting grains. For $\alpha=1$ the energy is obviously conserved.

In the presence of fragmentation, the number of particles will diverge. For $b_{c} \gamma_{0}-p_{0} / D>0$ the number of particles diverges asymptotically, while for $b_{c} \gamma_{0}-p_{0} / D<0$ this diver- gence takes place at a finite time, giving rise to shattering [30]. Everything else being constant, increasing $p_{0}$ increases the possibility of generating a shattering singularity. Since the total mass is conserved, the divergence in the number of particles implies that a dust is generated.

\section{Decreasing fragmentation probability}

In a freely evolving system, if the fragmentation probability vanishes asymptotically, the system will not develop any singularity in the number of grains. It evolves now as

$$
N=N_{0}\left[1+\left(\frac{E_{0}^{\rho}(\gamma / D+\rho)}{2 b_{c} \gamma_{0} \rho N_{0}^{\gamma / D+\rho}}\right)\left(1-e^{-2 b_{c} \gamma_{0} \rho \mathcal{C}}\right)\right]^{D / \gamma+\rho D},
$$

and for $\mathcal{C} \rightarrow \infty$ the system ends up with a finite number of grains, $N_{\infty}$ :

$$
N_{\infty}=N_{0}\left[1+\left(\frac{E_{0}^{\rho}(\gamma / D+\rho)}{2 b_{c} \gamma_{0} \rho N_{0}^{\gamma / D+\rho}}\right)\right]^{D / \gamma+\rho D} .
$$

In order to know the explicit time evolution, we need to solve for the number of collisions as a function of time as we have done before. The relation between the number of collisions and time now reads

$$
\frac{d \mathcal{C}}{d t}=N^{1 / D} v=N^{1 / D} E^{1 / 2} e^{-2 b_{c} \gamma_{0} \mathcal{C}} .
$$

For a large number of collisions, $\mathcal{C}$ tends asymptotically to

$$
\mathcal{C}=\frac{1}{\gamma_{0}} \ln \left[\frac{b_{c} \gamma_{0}\left(t-t_{0}\right)\left[1+\frac{E_{0}^{\rho}\left(\frac{\gamma}{D}+\rho\right)}{2 b_{c} \gamma_{0} \rho N_{0}^{\gamma / D+\rho}}\right]^{1 / \gamma / D+\rho}}{{ }_{1} \mathcal{F}_{2}\left(-\frac{1}{2}, \frac{1}{\gamma+\rho}, \frac{1}{2}, 0\right)}\right],
$$

and, accordingly, the total energy decays as

$$
E=\frac{E_{0}}{b_{c} t_{0}\left(t-t_{0}\right)^{2}} \frac{{ }_{1} \mathcal{F}_{2}^{2}\left(-\frac{1}{2}, \frac{1}{\gamma+\rho}, \frac{1}{2}, 0\right)}{\left[1+\frac{E_{0}^{\rho}(\gamma / D+\rho)}{2 b_{c} \gamma_{0} \rho N N_{0}^{\gamma+\rho+\rho}}\right]^{2 / \gamma / D+\rho}},
$$

where ${ }_{1} \mathcal{F}_{2}$ is a generalized hypergeometric function. Equation (29) has the same time dependence predicted by Haff. Such a result is not surprising given that asymptotically the number of cooling particles becomes constant.

For the singular case $\frac{\gamma}{D}+\rho=0$ and $\rho \neq 0$ the previous scenario does not change qualitatively. In this particular situation the number of grains evolves as

$$
N=N_{0} e^{E_{0}^{\rho} / 2 b_{c} \gamma_{0} \rho\left(1-e^{-2 b_{c} \gamma_{0} \rho \mathcal{C}}\right)},
$$

which for $\mathcal{C} \rightarrow \infty$ saturates to

$$
N_{\infty}=N_{0} e^{E_{0}^{\rho} / 2 b_{c} \gamma_{0} \rho} .
$$

The evolution equation for the number of collisions is simpler, leading to

$$
\mathcal{C}=\frac{1}{b_{c} \gamma_{0}}\left\{\ln \left[b_{c} \gamma_{0} E_{0}^{1 / 2} N_{0}^{1 / 2}\left(t-t_{0}\right)\right]\right\}
$$

Finally, the energy decays as 


$$
E(t)=\frac{1}{N_{0}\left[b_{c} \gamma_{0}\left(t-t_{0}\right)\right]^{2}},
$$

which recovers again the time dependence predicted by Haff.

\section{B. Kinetics of homogeneous steady states}

In order to reach a steady state with a constant mean kinetic energy, it is necessary to inject energy in the system. However, since the number of grains increases with time, the frequency of energy injection should also increase accordingly. Hence, $\psi \propto \omega(t)$. In this case the energy evolution can be written as

$$
\frac{d E}{d t}=-2 \gamma_{0} b_{c} \omega E+\xi^{2} \omega,
$$

leading to an asymptotic state characterized by a mean total energy

$$
E=\frac{\xi^{2}}{2 b_{c} \gamma_{0}}=E_{\infty} .
$$

As we have pointed out earlier, $\bar{v}^{2} \sim E$, implying that the mean velocity per particle does not evolve in time. As a result, the kinetics in these states is controlled only by the particle size. Specifically, the number of grains increases as

$$
\frac{d N}{d t}=N^{1 / D} v N^{-\gamma / D-\rho} v^{2 \rho} N,
$$

which in terms of $E_{\infty}$ reads as

$$
\frac{d N}{d t}=E_{\infty}^{\rho+1 / 2} N^{\kappa},
$$

with $\kappa=1+\frac{1}{D}-\frac{\gamma}{D}-\rho$. The general solution of Eq. (36) shows

$$
N=N_{0}\left[1-(\kappa-1)\left(t-t_{0}\right) E_{\infty}^{\rho+1 / 2} N_{0}^{\kappa-1}\right]^{1 / 1-\kappa} .
$$

For $\kappa<1$ the number of particles increases algebraically:

$$
N(t) \sim\left(t-t_{0}\right)^{1 / 1-k} .
$$

The form of the generalized Weibull breaking probability leads to different scenarios. If $\kappa<1$, at long times the number of grains increases algebraically as $N(t) \sim t^{1 /(1-\kappa)}$. On the contrary, for $\kappa>1$ a finite-time singularity at a shattering time $t_{s}$ develops and the number of grains in its vicinity diverges as $N(t) \sim\left(t_{s}-t\right)^{-1 /|\kappa-1|}$. The limiting situation $\kappa=1$ displays an exponential increase in the number of grains, which diverges at infinity.

In the present framework, a constant breaking probability corresponds to $\gamma=\rho=0$. If the breaking probability does not vanish, one always observes a shattering singularity and the number of grains in its vicinity diverges as $N \sim\left(t_{s}-t\right)^{-D}$. This situation corresponds to materials whose fragmentation probability does not depend significantly on the grain size and energy as it may happen for certain brittle materials [24]. Finally, for standard Weibull fragmentation distributions the breaking fragmentation is related to the density of surface defects. Accordingly $\gamma=D-1$ and $\rho>0$, and in this case the number of grains grows algebraically at long times, $N(t)$ $\propto t^{D /(D(\rho+1)-2)}$.
If energy injection scales generically with the collision frequency through an exponent $\zeta, \psi \propto \omega^{\zeta}$, then typically for $\zeta>1$ the granular gas heats up indefinitely, while for $\zeta<1$ it cools down. Depending on the details of the fragmentation process different kinetic scenarios are possible for both $\zeta$ $>1$ and $\zeta<1$.

\section{DIRECT SIMULATION MONTE CARLO TECHNIQUE}

A more detailed study of the kinetics of fragmenting gases requires the use of numerical analysis. To this end, we have adapted the direct simulation Monte Carlo technique, which has been widely used in the context of rarefied gases [31] and granular gases [23,32], and has been used recently to analyze fragmenting granular gases [11].

The assumption of spatial homogeneity simplifies the implementation of the DSMC technique, because it is enough to sample uniformly the relative orientation of colliding pairs $\hat{\varepsilon}$ without the need to keep track of particle positions. Sequentially, a pair of particles is selected at random. Then $\hat{\varepsilon}$ is chosen randomly on the unit sphere and the kinematic constraint is checked. In case the particles can collide, the cross section is computed and the collision takes place proportionally to its value. If the collision takes place, we need to decide if a colliding grain will subsequently break. To evaluate the fragmentation probability we estimate the stored elastic energy $E_{p}$ as the relative kinetic energy of the incoming colliding pair. On this basis we compute the fragmentation probability. If the fragmentation event is accepted, the grain breaks into two offsprings. During this process, mass, energy, and momentum are conserved. The collision frequency for a collision involving grains $i$ and $j$ can be computed on the basis of the cross section, and hence the time is advanced accordingly at each collision:

$$
t \rightarrow t+\frac{1}{N^{2}\left|v_{i j} \cdot \hat{\varepsilon}\right| \sigma_{i j}^{D-1}},
$$

where $N$ is the instantaneous number of grains.

In order to reach a steady state where the mean kinetic energy per particle is fixed, it is necessary to inject energy into the system. Although the kinetic evolution of the mean quantities is not sensitive to the details of the forcing mechanism, we will compare the implications of different thermostats on grain distribution functions. We describe first the different thermostats we have implemented.

\section{Energy injection: Thermostats}

We will analyze several procedures to input energy in a fragmenting dissipative gas. It is well known that for monodisperse granular gases the mechanism used to inject energy to reach a steady state determines the deviations observed from Gaussianity in the velocity distribution function $[23,33]$. The role of inelasticity on velocity statistics has been studied through Sonine expansions for different kinds of thermostats $[23,33]$. Numerical results have shown that the grain velocity distribution is by no means universal and depends on both the thermostat and grain interactions [33]. 
The simplest way to fix the mean kinetic energy of a particle system was introduced by Andersen [20], who proposed that particles collide with a virtual massive particle with frequency $\nu$. This procedure fixes the temperature of the particle to that of the thermostat, and in practice it means that the velocity of a particle is reassigned from a Gaussian with frequency $\nu$. Accordingly, in the interval $\Delta t$ a grain suffers a heating collision with probability $\nu \Delta t$. For a sufficiently high $\nu$ the velocity distribution corresponds to the Gaussian imposed by the bath; hence, it is possible to fix the temperature, although the system's dynamics is essentially controlled by the interaction with the thermostat.

In granular fluids, injecting energy homogeneously by applying a random force of prescribed amplitude with a given frequency $\nu$ has attracted the attention of many theorists [21,33-35]. If this heating frequency scales with the collision frequency, a steady state is reached in which the mean kinetic energy of the system does not change. Such a steady state arises as a competition between energy injection and energy dissipation. While Andersen fixes the temperature regardless of the properties of the system, this second energy injection mechanism will in particular lead to a diverging temperature for an elastic gas; hence, the temperature is a function of the inelasticity coefficient.

These two energy injection mechanisms conserve momentum only on average. One can enforce momentum conservation by subtracting the overall net momentum homogeneously over all particles. For a fragmenting system where particles of disparate sizes coexist, such a procedure can lead to large transient perturbations for the smallest species.

An alternative to the Andersen thermostat has been recently proposed by Lowe [22]. According to this LoweAndersen thermostat, with a frequency $\nu$ the relative velocity of a randomly chosen pair of particles is selected from a prescribed Gaussian. As was the case for the Andersen thermostat, also here the velocity distribution will obey a Gaussian distribution with the prescribed temperature imposed by the thermostat if the heating frequency is at least of the order of the collision frequency. The advantage of this thermostat is that momentum is conserved, and hence the dynamics of the grain velocity field will be properly accounted for; technically, we also avoid the problem of subtracting the centerof-mass acceleration and its impact on small-size grains. Specifically, for a randomly chosen pair of grains $i$ and $j$, their velocities are reassigned according to

$$
\mathbf{v}_{i}=\mathbf{V}_{\text {c.m. }}+\frac{\mu}{m_{i}} \frac{\mathbf{w}}{2}, \quad \mathbf{v}_{j}=\mathbf{V}_{\text {c.m. }}-\frac{\mu}{m_{j}} \frac{\mathbf{w}}{2},
$$

where $\mathbf{V}_{\text {c.m. }}=\left(m_{i} \mathbf{v}_{i}+m_{j} \mathbf{v}_{j}\right) /\left(m_{i}+m_{j}\right)$ is the center-of-mass velocity and $\mathbf{w}$ is a random velocity chosen from a prescribed Gaussian distribution.

A similar local generalization of the homogeneous random acceleration thermostat can be proposed: the relative homogenous forcing thermostat. With a fixed heating frequency $\nu$ a pair of particles is selected at random and a randomly chosen force with a prescribed mean amplitude, $F_{0}$, is applied to both particles in opposite directions. This local acceleration thermostat also preserves momentum conservation by construction and can therefore capture properly

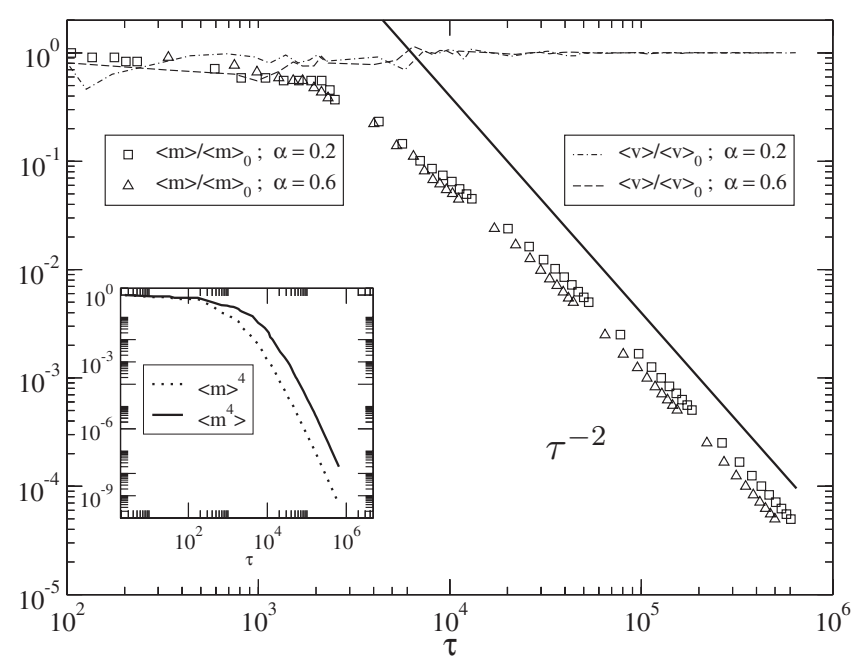

FIG. 1. Time evolution in a log-log scale of the mean size and characteristic velocities for different values of the restitution coefficient $\alpha$ and a constant fragmentation probability $p=1 / 100$. An auxiliary time scale $\tau$ is used to highlight the functional dependence, and the corresponding analytical power-law decays are displayed as solid lines. In the inset we display different moments of the mass distribution.

the dynamics of the collective variables of the system. Specifically, for a chosen pair of grains $i$ and $j$,

$$
m_{i} \mathbf{v}_{i}=m_{i} \mathbf{V}_{\text {c.m. }}+\mu \mathbf{g}+\mathbf{F}_{0}, \quad m_{j} \mathbf{v}_{j}=m_{j} \mathbf{V}_{\text {c.m. }}-\mu \mathbf{g}-\mathbf{F}_{0},
$$

where $\mu=m_{i} m_{j} /\left(m_{i}+m_{j}\right)$ is the reduced mass and $\mathbf{g}=\mathbf{v}_{i}-\mathbf{v}_{j}$ the relative velocity. We have verified that in the absence of fragmentation the velocity distribution functions already reported for homogeneous random forcing [21,23] are recovered.

\section{NUMERICAL RESULTS}

We will analyze the results obtained from DSMC simulations. We will validate the dynamic scaling scenario investigated theoretically in Sec. III and will subsequently describe the mass and velocity distributions which characterize the evolution of these fragmenting systems in the scaling regime. The numerical results are restricted to spatially homogeneous two-dimensional gases, $D=2$, of hard disks. In all the simulations we start from an equilibrium system of monodisperse disks and study their evolution after a transient once the system has lost memory of its initial state.

\section{A. Kinetics}

In order to validate the dynamic scaling regime, we have computed the total number of particles, $N(t)$ and the mean absolute velocity $\left\langle v^{2}\right\rangle^{1 / 2}$ as well as different moments of the particle size distribution, $\left\langle\sigma^{n}\right\rangle(t)$. If dynamic scaling holds, then $\left\langle\sigma^{n}\right\rangle \propto(\bar{r})^{n}$ and all the curves $\left\langle\sigma^{n}(t)\right\rangle^{1 / n}$ should become parallel to each other. For $D=2$, mass conservation ensures $\left\langle m^{1 / 2}\right\rangle=\langle\sigma\rangle \sim N^{-1}$. In Fig. 1 we display the time evolution of the quantities mentioned for a homogeneously forced granu- 


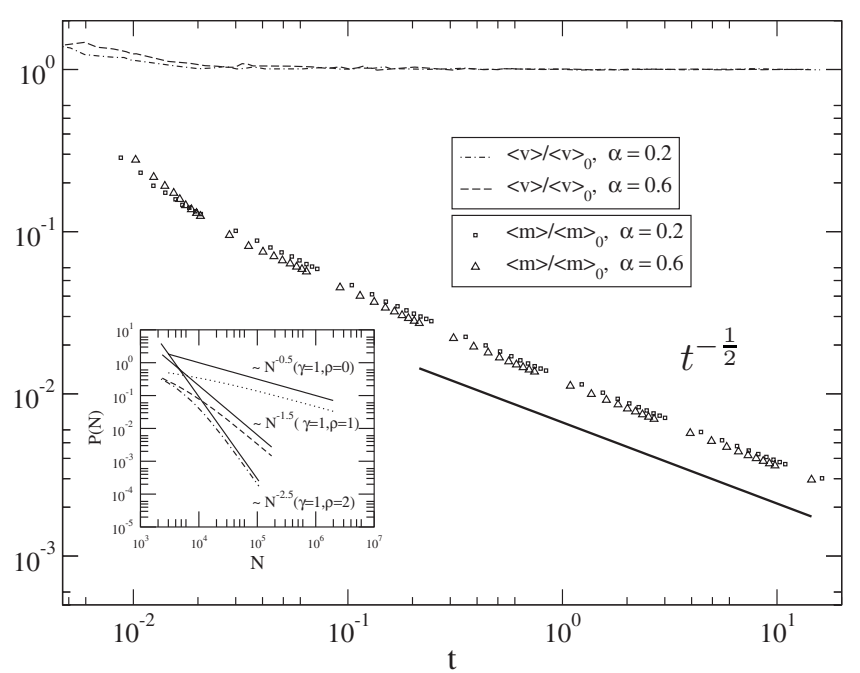

FIG. 2. Time evolution in a log-log scale of the mean size and characteristic velocity for different $\alpha$ values for a granular gas which breaks upon collision following a generalized Weibull breaking distribution, when it is heated through a local Lowe-Andersen thermostat. The inset shows the evolution of the mean breaking probability as a function of grain number [see Eq. (15)]. The corresponding analytical power-law decays are displayed as solid lines.

lar gas with different degrees of inelasticity, where grains break upon colliding with a constant probability $p=1 / 100$. Since a shattering divergence appears at a finite time, we use the auxiliary time scale $d \tau=\frac{N(t)}{N_{o}} d t$, introduced in Ref. [11]. In this intermediate scale, the divergence shifts to infinity, while the algebraic dependence of the typical size and velocity is preserved, although with modified exponents. For example, the mean mass decays as $\langle m\rangle \sim \tau^{-2}$ (see Sec. III B), a behavior that is clearly recovered in our numerical analysis of the Boltzmann equation as displayed in Fig. 1. In the figure one can appreciate the time scale in which the steady state is reached by tracing the variation of the mean grain velocity. A further validation of the dynamic scaling is shown in the inset, where we display several moments of the particle size distribution, which decay parallel to each other rather soon as the steady state is reached.

We have modified the inelasticity parameter and have observed always the same temporal dependence. Introducing a Lowe-Andersen thermostat does not lead to any significant deviation, consistent with the results which show that the behavior of the relevant size is only sensitive to the frequency of energy injection. We have also analyzed the kinetic evolution of a heated granular gas through a LoweAndersen thermostat when the fragmentation probability follows a generalized Weibull distribution, Eq. (1), in which defect formation is proportional to the grain surface $(\gamma=D$ $-1)$ and decreases continuously with time. In this case, the number of particles does not show any singularity, as can be appreciated from Fig. 2, which displays results for $\rho=2$. A dynamic scaling regime is achieved as soon as the system reaches its steady state. In the inset we show the time dependence of the fragmentation probability for different values of $\rho$ and compare them with their theoretical prediction. In the scaling regime an asymptotic algebraic decay of the frag-

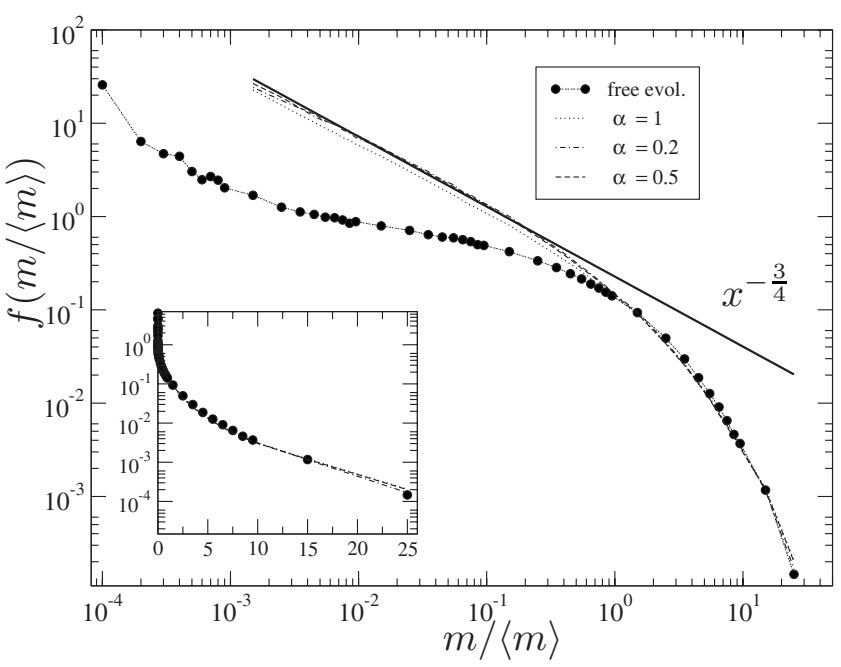

FIG. 3. Log-log plot of the scaled mass distribution of a granular gas with different degrees of inelasticity which break with a uniform probability $p=0.01$, when the system is heated through a Lowe-Andersen thermostat. We display as circles the freely evolving elastic granular system in the absence of energy injection. The solid line corresponds to a fitting for small sizes. The inset shows the same in semilogarithmic scale.

mentation probability is found. The results depicted are not modified if the thermostat and/or the degree of inelasticity are varied.

The results obtained with the DSMC technique validate the theoretical framework introduced in the previous section, according to which two different scenarios emerge in driven granular gases. If the fragmentation probability does not show a significant time dependence, then the number of grains will exhibit a finite time divergence characteristic of a shattering singularity. On the contrary, if the fragmentation probability decays algebraically to zero, so that it becomes less probable that grains of a given relative size break as time evolves, then the number of grains increases algebraically with an exponent related to the rate of decrease of the fragmentation probability.

\section{B. Particle mass distributions}

Grain fragmentation leads to a time evolving polydisperse mixture, and in the asymptotic dynamic scaling regime this polydisperse mixture changes only through the mixture mean particle size and velocity. In Fig. 3 we show results for the scaled mass distribution $f\left(\frac{m}{\langle m\rangle}\right)$, of a granular gas driven by a Lowe-Andersen thermostat which breaks with a constant breaking probability $p=1 / 100$. The distribution function shows an exponential decay at large sizes as displayed in the inset. However, for small particles the size dependence is consistent with an algebraic decay with exponent $-0.75 \pm 0.05$. This implies that there is no singularity in the mass distribution at small masses. We have observed that the scaled mass distribution is independent of the inelasticity parameter. However, a different behavior is observed for elastic fragmenting grains, which do not need a thermostat to reach a steady state. In this case the mass distribution is 


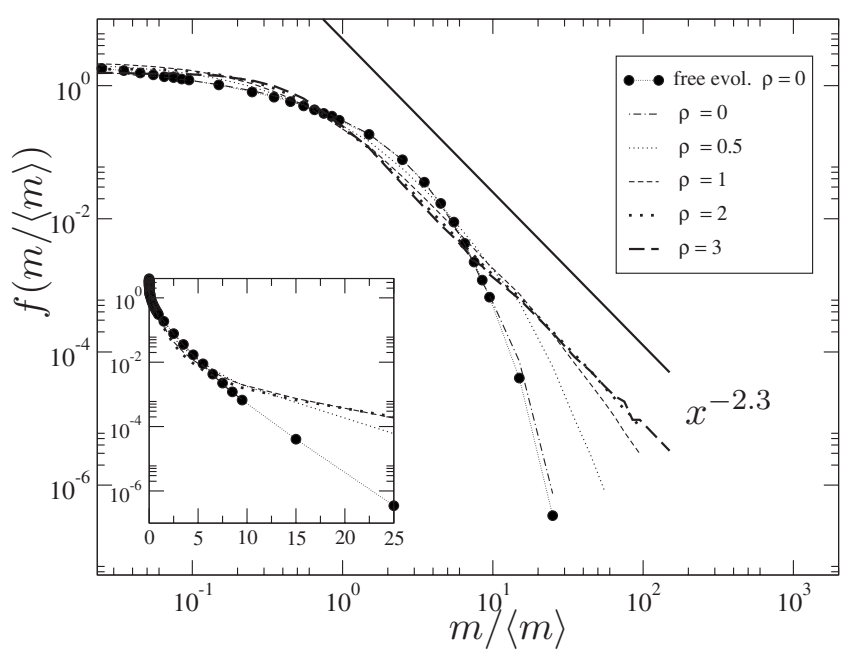

FIG. 4. Log-log plot of the scaled mass distribution of a granular gas with different degree of disorder $\rho$ according to a generalized Weibull distribution, as given in Eq. (1), with $\gamma=1$, when the system is heated through a relative homogeneous forcing thermostat. We display as circles the freely evolving granular gas. The solid line corresponds to a fitting for large sizes.

similar to the one described for large and intermediate masses, but displays a qualitatively different behavior from that of inelastic grains in the region of small particles. It is in fact characterized by the formation of extremely small grains: a powder. This powder signals the existence of a fragmentation process [6], in which the small fragments tend to follow a sequence of breaking events, due to the high velocity of the smaller particles.

We can also apply the Lowe-Andersen thermostat to the elastic gas. In this case the system is brought to a steady state where the temperature is fixed by the thermostat. The mass distribution shows a qualitative change and becomes essentially identical to that of inelastic grains, which displayed an algebraic decay at small masses. This fact shows that inelasticity is not as relevant, as it would seem at first sight, and that the velocity distribution plays a relevant role in the process of collision-induced fragmentation, as we will discuss in the next subsection. If a local homogeneous random forcing thermostat is applied to inelastic gases, the scaled mass distributions recovered are essentially equivalent to the ones shown in Fig. 3.

We display in Fig. 4 the scaled mass distributions of a heated granular gas which fragments following a generalized Weibull distribution. We consider a local forcing thermostat and analyze the impact of modifying the shape of the fragmentation probability distribution by changing $\rho$. For small particles the mass grain distribution becomes uniform. In fact, the smaller the grains, the less likely they will break; as a result, they evolve more slowly than their larger counterparts. The latter induce a more uniform distribution of sizes which might be associated with the development of an effective threshold at small sizes. In the limiting case when the fragmentation probability does not depend on grain velocity, $\rho=0$, the large mass tail is consistent with an exponential. As $\rho$ increases, there is an asymptotic departure toward an algebraic behavior characterized by an exponent $-2.3 \pm 0.05$ in-

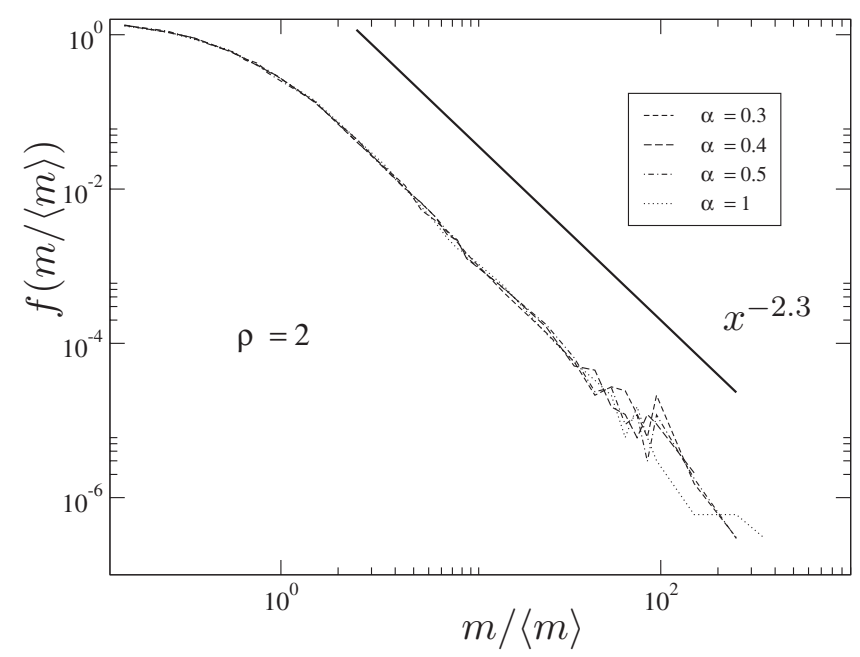

FIG. 5. Log-log plot of the scaled particle mass distribution for a granular gas which breaks following a generalized Weibull fragmentation probability with $\rho=2$ and different degrees of inelasticity. The system reaches a nonequilibrium steady state using a LoweAndersen thermostat. The corresponding fitted power-law decay appears as a solid line.

dependent of $\rho$. However, with the current data it is not possible to determine whether this algebraic behavior is indeed the true asymptotic regime.

We have considered a system of elastic grains in the limiting case $\rho=0$ and have observed that for a vanishing fragmentation probability the mass distribution does not show the deviations reported in Fig. 3. If a Lowe-Andersen thermostat is used instead of local relative forcing, the scaled mass distributions are not significantly altered. Finally, Fig. 5 shows the independence of the mass distributions on the inelasticity parameter for a generalized fragmentation Weibull distribution with parameter $\rho=2$, when a Lowe-Andersen thermostat is considered. One can clearly see that the inelasticity does not affect the relative distribution of masses and that in the elastic limit no deviations are observed.

\section{Particle velocity distributions}

As we have pointed out earlier, in the asymptotic dynamic scaling regime the polydisperse mixture which characterizes the fragmenting gas evolves only through the mean particle size and velocity. For a freely evolving elastic gas which breaks with constant probability, large deviations from a Maxwellian distribution are observed because smaller particles acquire a proportionally larger velocity, as depicted in Fig. 6(a). This marked deviation can be traced to the asymmetric dynamic behavior of small and large grains, as shown in the inset of Fig. 6(a). It becomes clear that the maximum in the grain velocity distribution at a finite velocity comes from smaller grains, a behavior analogous to the one observed previously for freely evolving nearly elastic grains [11]. The characteristic velocities of the smallest and largest fractions of the grains differ significantly.

The functional form of the velocity distribution depends on the fragmentation process; for a generalized Weibull fragmentation probability, the asymmetric behavior of small and 

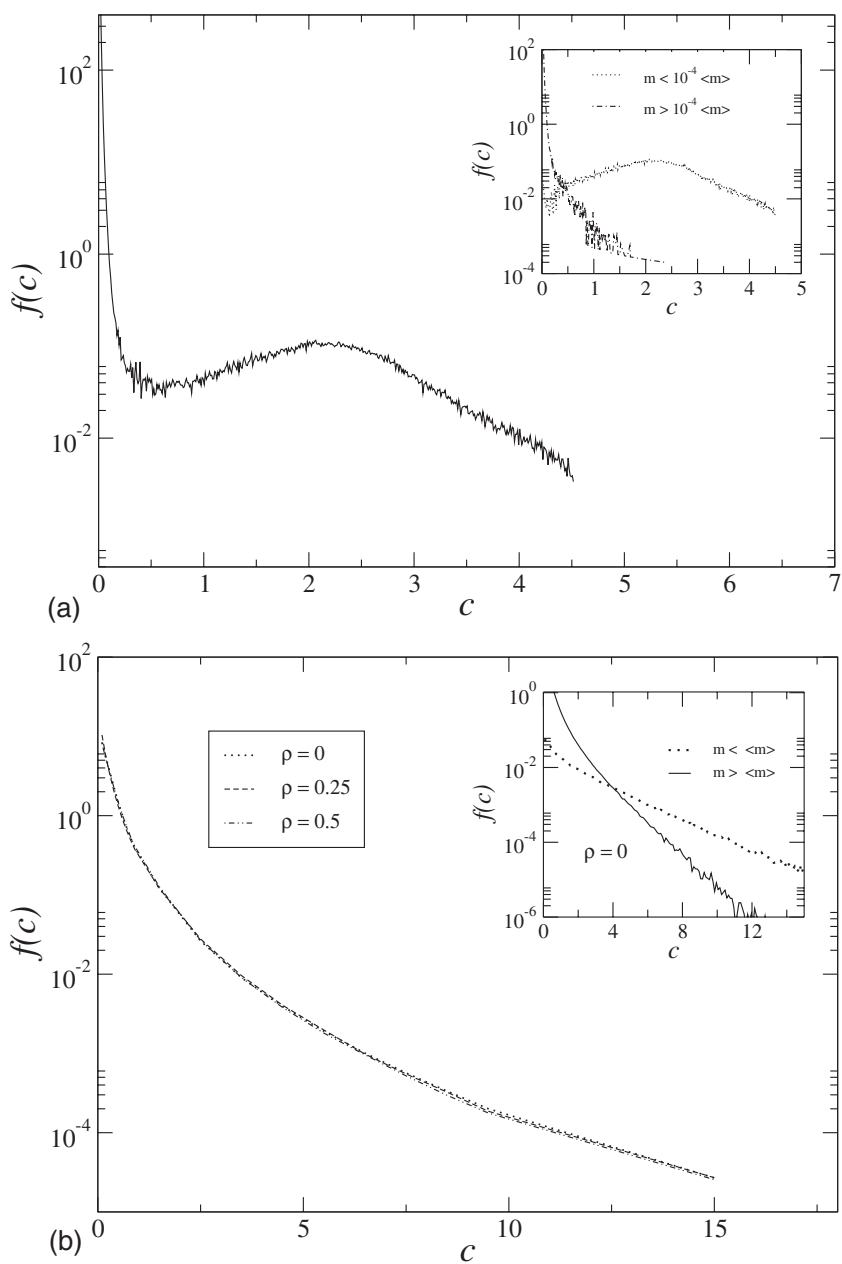

FIG. 6. Log-log plot of the scaled particle velocity distribution for an elastic fragmenting gas. (a) Constant fragmentation probability $p=0.1$. (b) Generalized Weibull fragmentation probability $\gamma=1$ and varying $\rho$. In both figures, the insets show the scaled velocity distributions for small $m<m^{*}$ and large $m>m^{*}$ grains. For convenience, a different characteristic value $m^{*}$ has been used in each case.

large grains is lost [see Fig. 6(b)]. It is noticeable that during fragmentation the generation of very fast and light particles is again detected. However, in the scaling regime the effective breaking probability decreases as the number of particles within the system increases, as can be appreciated in Eq. (15). This decrease prevents the formation of a dust of small grains.

The mechanism of energy injection does affect the shape of the velocity distribution, even in the absence of fragmentation due to the breakdown of detailed balance $[23,33,36]$. In general, overpopulated high-energy tails with asymptotic decay $f(c) \sim \exp \left[c^{n}\right]$ are obtained. Hence, power-law and Gaussian tails correspond to borderline cases [33,36]. An example of the latter is obtained with a Lowe-Andersen thermostat at high injection frequency [37]. For momentumconserving thermostats, we have analyzed the velocity distributions obtained in the steady states in the absence of fragmentation. In Fig. 7 the scaled velocity distributions obtained for several frequencies of energy input are displayed.

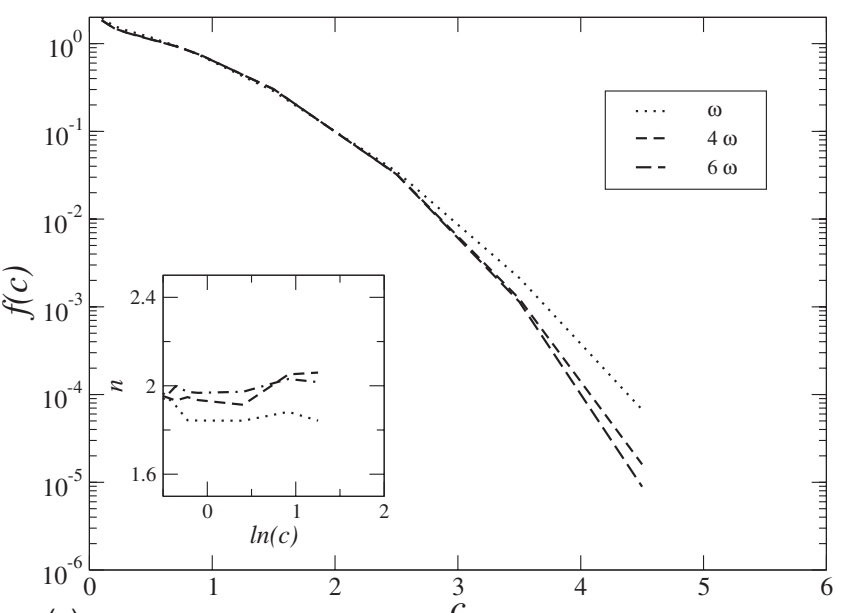

(a)

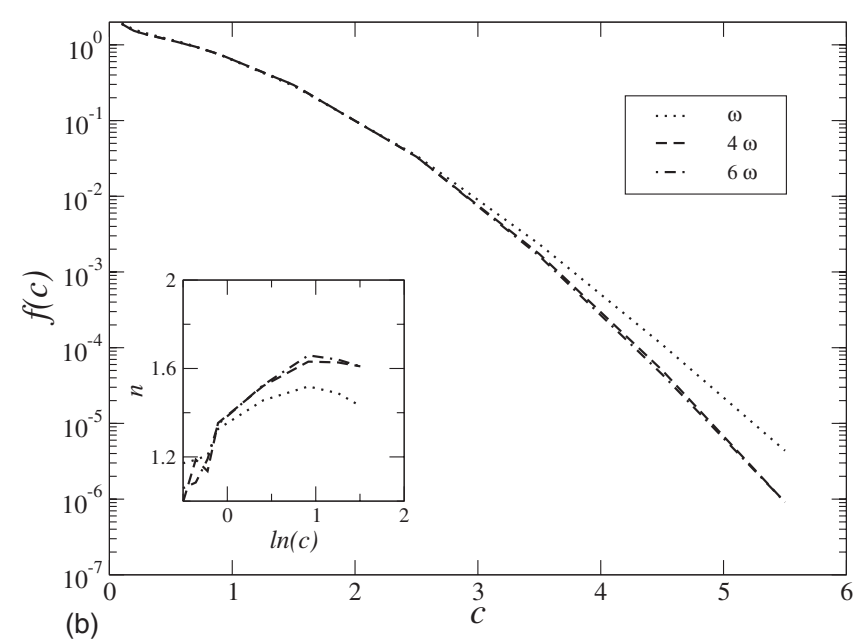

FIG. 7. Linear-log plots of the scaled velocity distributions for a monodisperse granular gas driven by (a) a Lowe-Andersen thermostat and (b) local relative forcing thermostat. The insets show the exponent $n$ which characterizes the asymptotic decay of the velocity distributions.

In order to assess the effect of the thermostat on the asymptotic, large velocity decay, we assume a functional form $f(c) \sim A \exp \left[-B c^{n}\right]$ and estimate the exponent $n$ using the infinite limit of the corresponding logarithmic derivative:

$$
n=\lim _{c \rightarrow \infty} \frac{\partial}{\partial \ln c} \ln \left[\ln \left(\frac{f(0)}{f(c)}\right)\right] .
$$

In the insets of Figs. 7(a) and 7(b) we show the estimated exponents for Lowe-Andersen and local acceleration thermostats. The Lowe-Andersen thermostat gives rise to asymptotic tails consistent with a Gaussian $n=2.05 \pm 0.05$, independently of the heating frequency when the number of heating events suffered by a grain between collisions is large. For the local acceleration method, we obtain $n=1.6 \pm 0.05$, which is consistent with the same decay $f(c) \sim \exp \left(c^{-3 / 2}\right)$ as predicted for the already studied random forcing thermostat $[21,23]$. The mean values and the confidence intervals are estimated using the $n$ data values for $c>1$ in the highfrequency limit. 


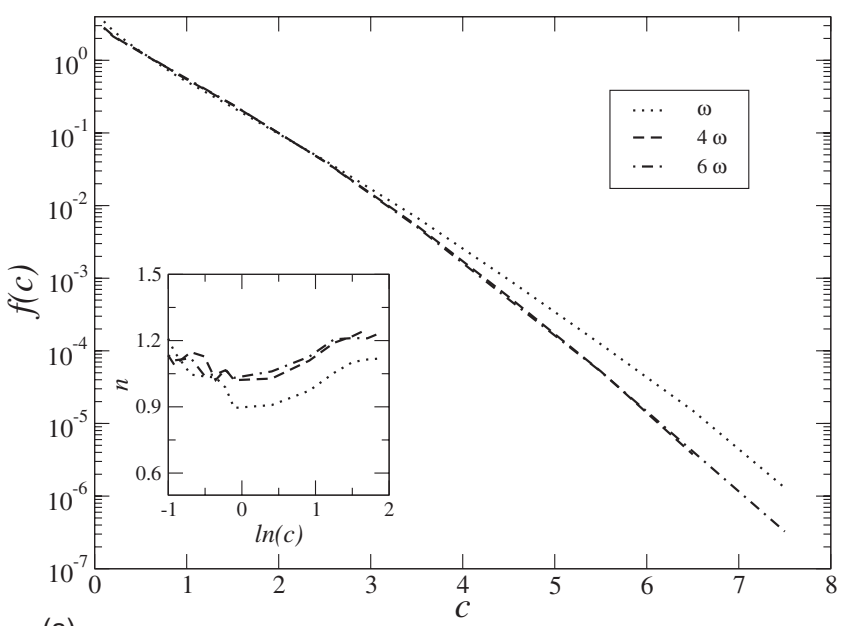

(a)

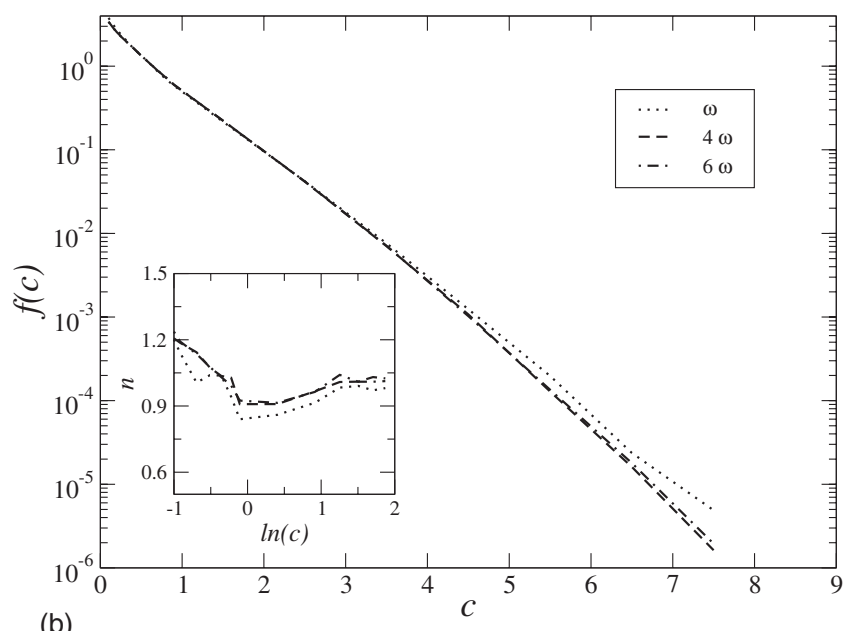

(b)

FIG. 8. Linear-log plot of particle velocity distributions, scaled by the mean velocity. Results for granular gases which break with a constant fragmenting probability $p=1 / 10$, driven by two different heating mechanisms: (a) Lowe-Andersen thermostat and (b) relative homogeneous forcing thermostat. The insets show the exponent $n$ which characterizes the asymptotic decay of the velocity distributions. Different heating frequencies are shown.

Collision-induced grain fragmentation interferes significantly with the grain velocities and leads to significant departures in the velocity distribution function with respect to their counterparts in the absence of grain breakup. In Figs. 8(a) and 8(b) we show results for granular gases, which break with a constant fragmenting probability. Additionally, Figs. 9(a) and 9(b) illustrates the scaled velocity distributions of fragmenting granular gases which break through a generalized Weibull distribution. For each case, two energy injection mechanisms have been explored. The data in Figs. 8(a) and 9(a) correspond to a local Lowe-Andersen thermostat, while Figs. 8(b) and 9(b) show the outcomes obtained from systems in contact with a local acceleration thermostat. The insets of the figures display the value of the exponent $n$, assuming that the distribution follows a generalized exponential decay $f(c)=A \exp \left(-B c^{n}\right)$.

For the Lowe-Andersen thermostat [Figs. 8(a) and 9(a)], independently of the breaking mechanisms, the scaled veloc-
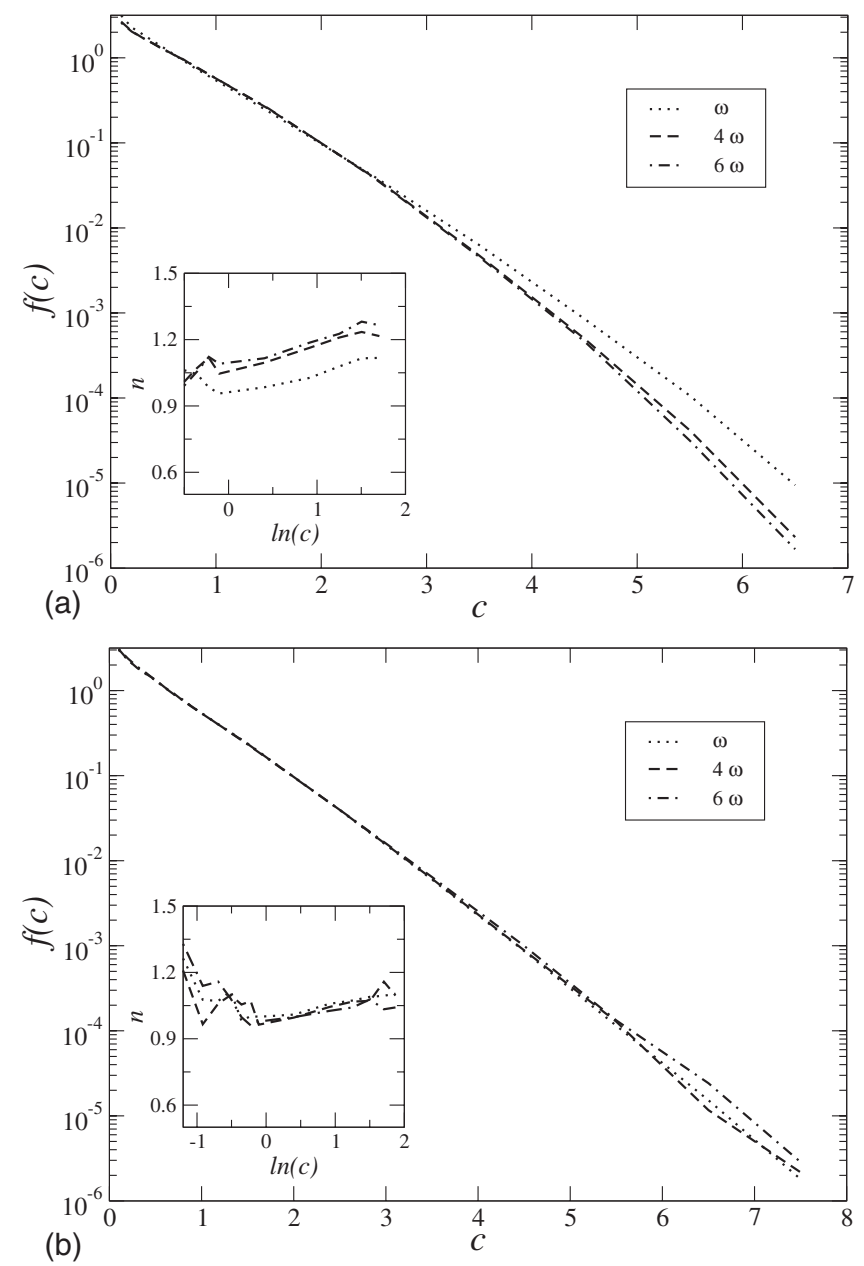

FIG. 9. Linear-log plots of the scaled velocity distributions for fragmenting granular gases which break through a generalized Weibull distribution with $\rho=2$. The gas is driven to a steady state using different thermostats: (a) Lowe-Andersen and (b) relative homogenous forcing. The insets show the exponent $n$ which characterizes the asymptotic decay of the velocity distributions. Different forcing frequencies are displayed.

ity distributions are compatible with generalized exponential decays $\exp \left(-c^{n}\right)$. In the case of fragmenting systems with constant breaking probability the decay has been consistent with $n=1.20 \pm 0.05$, and for velocity-dependent breaking rules the asymptotic exponents numerically are $n$ $=1.15 \pm 0.05$. The reported values correspond to the limit where a grain, on average, suffers a large number of heating events between collisions.

For the local acceleration thermostats [Figs. 8(b) and 9(b)] the velocity distributions are more robust to changes in the heating frequency. Given the numerical precision, the changes are always minor and we can consider that essentially the decay is exponential $n \approx 1$ regardless of the heating frequency.

In general, we observe a very weak dependence of the shape of the distribution on the breaking rule. Moreover, for the ranges explored the shape of the velocity distribution is independent of the heating frequency. All numerical evidence strongly supports that in driven fragmentation the scaled velocity distribution follows a generalized exponential behav- 

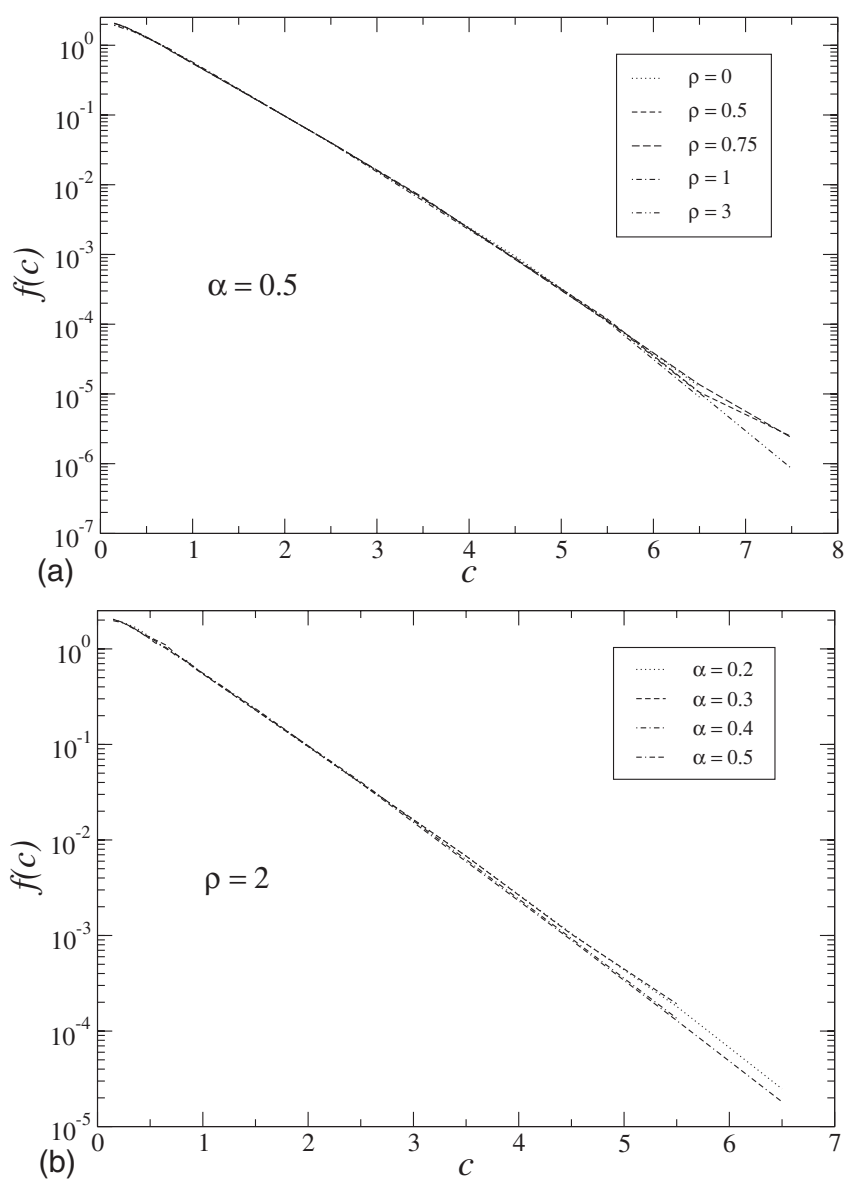

FIG. 10. Linear-log plot of particle velocity distributions, scaled by their mean velocities. Results (a) for particles with different breaking probabilities, Eq. (1), changing $\rho$ and keeping $\alpha=0.5$, and (b) several inelasticities are illustrated with $\rho=2$.

ior $f(c) \sim \exp \left(-c^{n}\right)$, where the exponent $n$ is fairly insensitive to the fragmentation mechanisms. In Fig. 10 we show also that both the degree of grain inelasticity and the exponent which characterizes the Weibull distribution do not affect significantly the grain velocity distributions.

Finally, the velocity distributions of a subset of grain species (data not shown) show different widths and mean values. This cast doubts on the meaning of a single temperature to characterize fragmenting polydisperse granular mixtures. The absence of a common temperature invalidates the use of perturbative calculations-e.g., through a Sonine expansion-to determine the deviations from Gaussian behavior. The existence of a stationary size distribution suggests the possibility to map the fragmenting granular gas onto an effective polydisperse mixture with a time-dependent mean. Although this is indeed possible in principle, we do not have any simple theoretical framework to predict either the power-law decays or the details of the tails of $f\left(\frac{m}{\langle m\rangle}\right)$ and $f(c)$.

\section{CONCLUSIONS}

We have studied the kinetics of granular gases which can fragment upon collision. We have shown that generically the gas evolves at long times to a dynamic scaling regime, in which its properties are controlled by the temporal evolution of the mean grain size and velocity. We have seen that the interplay between collisions and fragmentation leads to strong correlations in the temporal behavior of the fragmenting gases. Therefore, the kinetics in these materials arises as a balance between these two processes. This fact shows that collision-induced fragmentation imparts the system with properties which are different from other systems in which the fragmentation depends on the particle sizes, but not on their velocities, such as in the standard mean-field theory of fragmentation. This result is complementary to the observation in coagulation systems, where also the correlations between the velocities of the colliding pair and their structural properties determining the likelihood of aggregating produce kinetic scenarios which differ from those based on Smoluchowski approaches [38]. We have performed a wide study, in which we have varied the grain fragmentation probability, the inelasticity of the grains, and the way in which energy is imparted in the system, although we have restricted ourselves to homogeneous gases. We have implemented different thermostats and have in particular proposed a new forcing thermostat which conserves momentum locally.

We have obtained analytic results for the time evolution of the number of grains in the asymptotic regime and have shown that it depends both on the gas properties and the details of the fragmentation process. Although we have considered that fragmentation is determined by a generalized Weibull distribution, the analysis has shown that the granular gas evolution is controlled by the asymptotic behavior of the effective fragmentation probability. If the fragmentation probability is constant or tends asymptotically to a constant value, then the number of grains diverges at a finite time; a shattering singularity develops and the system will form a dust. On the contrary, if the mean breaking probability tends algebraically to zero, the divergence at finite time is generically not present. In this respect, the results obtained are generic for any fragmentation probability which vanishes analytically. In both cases information on the breaking kinetics can be induced from the temporal increase in the grain number and opens an alternative procedure to study the details of the fragmentation process in granulate materials. In the scaling regime, we have also analyzed mass and velocity distributions. We have seen that the fragmentation process plays a relevant role in the shape of the mass distribution and that it also interferes with the velocity distribution of the particles. When the fragmentation is driven by local stochastic events, we have shown that the long velocity tail is essentially exponential independently of the heating frequency and the details of the breaking process. On the contrary, the Lowe-Andersen thermostat leads to a scaled velocity distribution which follows a generalized exponential behavior $f(c) \sim \exp \left(-c^{n}\right)$, with $n \approx 1.2$.

The results obtained generalize previous studies on freely cooling fragmenting gases, but are still restricted to spatially homogeneous systems. The insight gained will be relevant to address the kinetics of similar systems under inhomogeneous drivings, which are more relevant from the understanding of transport and handling of granular materials. 


\section{ACKNOWLEDGMENTS}

R.C.H. acknowledges the financial support of the Spanish Minister of Education. I.P. and R.C.H. thank the Spanish
M.E.C. (Grant No. FIS2005-01299) and I.P. also acknowledges DURSI (Distinció de la Generalitat de Catalunya) (Spain) for financial support. The authors thank M. H. Ernst and E. Trizac for fruitful discussions and suggestions.
[1] Z. Grof, J. Kosek, M. Marek, and P. M. Adler, AIChE J. 49, 1002 (2003).

[2] A. Weber, U. Teipel, and H. Nirschl, Chem. Eng. Technol. 29, 642 (2006)

[3] T. Jiang and J. A. Northby, Phys. Rev. Lett. 68, 2620 (1992).

[4] L. Oddershede, P. Dimon, and J. Bohr, Phys. Rev. Lett. 71, 3107 (1993).

[5] D. Nesvorny, D. Vokrouhlicky, W. F. Bottke, and M. Sykes, Icarus 181, 107 (2006).

[6] S. Redner, in Statistical Models for the Fracture of Disordered Media, edited by H. J. Herrmann and S. Roux (Elsevier, Amsterdam, 1990).

[7] J. A. Âström, Adv. Phys. 55, 247 (2006).

[8] M. H. Ernst and I. Pagonabarraga, J. Phys. A 40, F331 (2007).

[9] P. L. Krapivsky and E. Ben-Naim, Phys. Rev. E 68, 021102 (2003).

[10] F. Spahn, N. Albers, M. Sremcevic, and C. Thornton, Europhys. Lett. 67, 545 (2004).

[11] I. Pagonabarraga and E. Trizac, in Theory of Granular Gas Dynamics, edited by T. Pöschel and N. Brilliantov (SpringerVerlag, Berlin, 2003).

[12] J. J. Gilvarry and B. H. Bergstrom, J. Appl. Phys. 32, 400 (1961).

[13] T. Ishii and M. Matsushita, J. Phys. Soc. Jpn. 61, 3474 (1992).

[14] A. Meibom and I. Balslev, Phys. Rev. Lett. 76, 2492 (1996).

[15] A. Diehl, H. A. Carmona, L. E. Araripe, J. S. Andrade, Jr., and G. A. Farias, Phys. Rev. E 62, 4742 (2000).

[16] L. E. Araripe, J. S. Andrade, Jr., and R. N. Costa Filho, Phys. Rev. E 71, 036119 (2005).

[17] V. Buchholtz, J. A. Freund, and T. Pöschel, Eur. Phys. J. B 16, 169 (2000).

[18] J. A. Âström and H. J. Herrmann, Eur. Phys. J. B 5, 551 (1999).
[19] R. C. Hidalgo and I. Pagonabarraga, Europhys. Lett. 77, 64001 (2007).

[20] Hans C. Andersen, J. Chem. Phys. 72, 2384 (1980).

[21] T. van Noije and M. Ernst, Granular Matter 1, 57 (1998).

[22] C. P. Lowe, Europhys. Lett. 47, 145 (1999).

[23] Jose Maria Montanero and Vicente Garzó, Granular Matter 4, 17 (2002).

[24] E. W. Andrews and K. S. Kim, Mech. Mater. 29, 161 (1998).

[25] F. Kun and H. J. Herrmann, Phys. Rev. E 59, 2623 (1999).

[26] Bikas K. Chakrabarti and L. Gilles Benguigu, Statistical Physics of Fracture and Breakdown in Disordered Systems (Oxford University Press, New York, 1997).

[27] Z. Cheng and S. Redner, J. Phys. A 23, 1233 (1990).

[28] S. McNamara and W. R. Young, Phys. Fluids 5, 34 (1993).

[29] P. K. Haff, J. Fluid Mech. 134, 401 (1983).

[30] E. D. McGrady and R. M. Ziff, Phys. Rev. Lett. 58, 892 (1987).

[31] G. Bird, Molecular Gas Dynamics and the Direct Simulation of Gas Flows (Clarendon, Oxford, 1994).

[32] V. Garzo and A. Santos, Kinetic Theory of Gases in Shear Flows. Nonlinear Transport (Kluwer Academic, Dordrecht, 2003).

[33] M. H. Ernst and R. Brito, Europhys. Lett. 58, 182 (2002).

[34] I. Pagonabarraga, E. Trizac, T. P. C. van Noije, and M. H. Ernst, Phys. Rev. E 65, 011303 (2001).

[35] D. R. M. Williams and F. C. MacKintosh, Phys. Rev. E 54, R9 (1996).

[36] M. H. Ernst and R. Brito, Phys. Rev. E 65, 040301(R) (2002).

[37] A. Barrat, T. Biben, Z. Ràcz, E. Trizac, and F. van Wijland, J. Phys. A 35, 463 (2002).

[38] E. Trizac and P. L. Krapivsky, Phys. Rev. Lett. 91, 218302 (2003). 